\title{
Change Detection in Vital Signs Associated with Impending Death for Homecare Patients Using a Pressure-Sensing Mat
}

\author{
Takayuki Shuku*, Noriko Sakano², Mizuki Morita² and Shingo Kasahara ${ }^{2}$ \\ ${ }^{1}$ Graduate School of Environmental and Life Science, Okayama University, Okayama, Japan \\ ${ }^{2}$ Graduate School of Medicine, Dentistry, and Pharmaceutical Sciences, Okayama University, Okayama, Japan
}

\begin{abstract}
How vital signs change at the end of life is a frequently asked question by health-care professionals, patients, and families, and research groups have attempted to identify specific changes and indicators associated with impending death. This study proposed a new method for detecting characteristic changes in vital signs associated with patient's death based on time-series data measured by a pressuresensing mat. The pressure-sensing mat is less invasive and can automatically log heart and respiratory rates with high temporal resolutions. In the proposed method, the probability density functions (PDFs) of vital signs are estimated based on the large amount of data, and temporal
\end{abstract}

changes in the PDFs are evaluated using an information measure, the Jenson-Shannon divergence (JSD). The proposed method was used to detect changes in vital signs of homecare patients to demonstrate its applicability. By tracking the temporal changes in the PDFs and JSD of vital signs, the specific changes associated with death can be detected. The proposed method can be a promising approach in quantitative and simplified prediction of impending death in homecare patients.

\section{Keywords}

Vital signs; Pressure-sensing mat; Change detection; Probability density functions

\section{Correspondence to:}

\section{Takayuki Shuku}

Graduate School of Environmental and Life Science, Okayama

University, Okayama, Japan.

E-mail: shuku@cc.okayama-u.ac.jp

\section{EJBI 2018; 14(1):52-57}

Received: December 28, 2017

Accepted: February 13, 2018

Published: February 20, 2018

\section{Introduction}

Clinicians observe decline in physical function (or activities of daily living, ADLs) in patients at the end of their lives, and patients experience a multitude of physiological changes such as muscle weakness, dysphagia, and altered level of consciousness $[1,2]$. In addition to these physiological changes, vital signs such as heart rate, blood pressure, and respiratory rate also provide useful information on the patient's health status, and these changes can signify impending death [3]. Accurate prediction of impending death is of great importance to clinicians who attend to patients at the end of life because it could affect their communication with patients and families and inform complex decision making such as discontinuation of investigations and aggressive treatments, discharge planning, and enrollment into clinical care pathways.

Several studies have examined diagnostic signs of impending death. Bruera et al. [4] investigated variation in vital signs in the last days of life in patients and reported that blood pressure and oxygen saturation decreased as patients approach death. Hui et al. [5] identified eight highly specific physical signs associated with death within 3 days among cancer patients. The published studies, however, were designed for hospitalized patients with advanced cancer, and the applicability of their findings to homecare patients is extremely limited. Development of simplified methodologies to detect the changes associated with impending death for homecare or non-cancer patients is necessary. Some research groups have developed methods of predicting death in homecare patients, and their predictions have performed well [6, 7, 8]. Many existing methods, however, were developed to predict relatively long survival times (e.g., up to 10 years). Long-term predictions have limited application in immediate care planning and decision making, especially for homecare patients, who may be in their final days of life, and their families. In addition, most existing prognostic methods are not adaptive and were not developed as web applications. 
Recently, sophisticated sensors designed to measure vital signs have been developed, and a pressure-sensing mat has been paid great attention because it is less invasive and can automatically measure heart and respiratory rates with high temporal resolution. It would be a promising approach to identify characteristic changes associated with impending death based on large amounts of data measured by the pressure-sensing mats. A number of studies have developed less/non-invasive monitoring using pressure sensors $[9,10,11]$. These sensors are basically designed to evaluate sleep quality or to automatically detect sleepwake states, but they have never used for predicting impending death for patients [12] outlined how future health care is shaping up current research efforts in devices and sensors, signal processing, and machine learning and reported the potential of sensor data-driven prognosis.

This study proposes a new methodology to detect the changes in vital signs associated with impending death based on the hightemporal-resolution data measured by a pressure-sensing mat and to analyze the data using a machine learning method, i.e., density ratio estimation [13]. This paper describes the concept of the method proposed in this study and the application example for actual vital signs measured by a pressure-sensing mat.

\section{Materials and Methods}

\subsection{Sensor Mat}

We used the sensor mat Odayaka Time developed by FSC Co., Ltd. (Nagoya, Japan) to measure time-series vital signs for homecare patients (shown below). This sensing mat can be placed on the top of any mattress and measure pressure based on the patient's body motion in bed. Then, vital signs are estimated based on the measured pressure. The mat continuously takes measurements and is flexible, thin, and unnoticeable.

The sensor does not properly work when the patient's back is off the mat and sometimes cannot measure data. We defined the missing data rate and used it as an auxiliary data for detecting changes in patient's status.

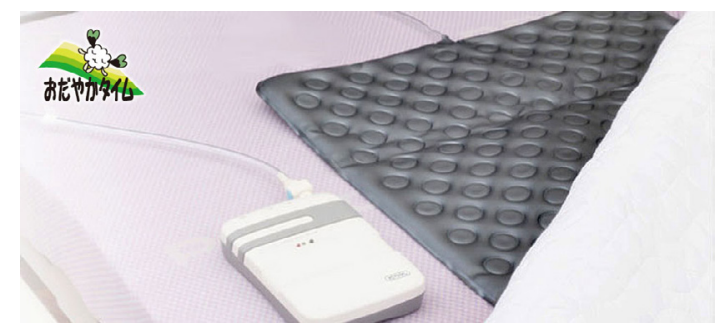

Pressure-sensing mat: Odayaka Time.

\subsection{Data Collection}

Although 180 patients have been equipped with this mat, we only collected time-series data from two bedridden homecare patients, patients 1 and 2, whose vital signs were measured until death. This is because the other data are not consistent and appropriate for the analysis. Information about the two patients is summarized in Table 1 . The measurement period for patients 1 and 2 was 8 and 3 months, respectively. The patient requirement and study design were approved by the ethics committee of Okayama University, and informed consent was obtained from the patients or their families.

\subsection{Data Analysis based on Density Ratio}

\section{Estimation}

We developed a new method for detecting changes in time-series vital signs using density ratio estimation [13]. The methods for change/anomaly detections in timeseries data include dynamic time-warping method (TWM) [14], singular spectrum transformation (SST) [15], and autoregressive (AR) model-based method [16]. These methods, however, have some drawbacks in practical applications, such as sensitivity to noise (TWM), time consuming (SST), and inability to detect drastic changes in time-series data (AR model-based method). Recently, the more advanced method of change detection, called density ratio estimation, has received much attention because of its simplicity and robustness and its application in time-series data analysis [13].

To develop the new method, we made the following assumptions:

1. The value of the vital sign is a sample generated from the inherent probability density functions (PDFs) of the patients.

2. Change in the PDFs shows the patient's health status.

3. Specific changes in vital signs associated with impending death can be detected by determining the temporal change in the PDFs.

The PDFs of the heart and respiratory rates are estimated based on the time-series data measured by the pressuresensing mat. The histogram of the vital signs was drawn using the data in specific time intervals ( 24 hours in this study). Since the temporal resolution of the data is 1 minute, one PDF is represented by 1,440 discrete samples. We applied the Jenson-Shannon divergence (JSD) to evaluate quantitatively the temporal change in the PDFs because it is a finite value, compared with other information divergence such as Kullback-Leibler divergence (KLD). The JSD is given by:

$J S D\left(p_{t} \mid p_{t-1}\right)=\frac{1}{2} K L D\left(p_{t} \mid q\right)+\frac{1}{2} K L D\left(p_{t-1} \mid q\right)$

where $p$ indicates the PDF of vital signs and its subscript indicates time; $q$ and KLD are defined as: 
Table 1: Patient information.

\begin{tabular}{lll}
\hline & Patient 1 & Patient 2 \\
\hline Sex & Female & Male \\
Age (years) & 94 & 100 \\
Disease & Cerebral infarction & Pneumonia \\
\hline
\end{tabular}

$$
\begin{gathered}
q=\frac{1}{2}\left(p_{t}+p_{t-1}\right) \\
K L D\left(p_{t} \mid p_{t-1}\right)=\sum_{i} p_{t}(i) \log \frac{p_{t}(i)}{p_{t-1}(i)} .
\end{gathered}
$$

When the two PDFs, $p_{\mathrm{t}}$ and $p_{t-1}$, are more similar, the JSD decreases. By determining the temporal change in the JSD, we can identify the characteristic changes in the patient's status.

\section{Results}

\subsection{Temporal Changes in the PDFs of Vital Signs}

Figure 1 shows the temporal changes in the PDFs of the heart and respiratory rates of patients 1 (a) and 2 (b), with the horizontal and vertical axes indicating the elapsed time and vital signs, respectively. The color shows the probability or frequency of the data. Figure 2 explains the interpretation of Figure 1, where the three continuous lines indicate the probability functions with different variance (large, medium, and small) and the colored figures next to each probability function indicate each probability function represented by a color map. In the color maps, the reddish and bluish colors represent higher and lower probabilities, respectively. Thus, the changes in the trend (the most probable values) and variance in time-series data can be easily visualized. In patient 1 (Figure 1 (a)), the most probable value of the heart rate fluctuated from 40 to 70 days and then remained constant after 100 days. Regarding the respiratory rate, although the PDF had large variance until 100 days, the variance decreased after 110 days. Figure 3 compares the PDF before 110 days (60 days) with that after 110 days (160 days). Using the PDFs, represented by color maps, the one-dimensional PDF is easily visualized. Although the PDF at 60 days did not have any peaks and had larger variance, the PDF at 160 days showed typical Gaussian distribution with one peak. These color maps enabled the visualization of the temporal change in the PDFs, making it easy to confirm the changes. In patient 2, although both vital signs fluctuated slightly until 90 days, the most probable values drastically increased just 7 days before death. This drastic increase in vital signs can be used as an indicator for impending death.

\subsection{Temporal Changes in the JSD and Missing Data Rate}

The temporal change in the JSD of patients 1 and 2 are shown in Figures 4 and 5, respectively. The JSD value is a measure normalized between 0 and 1 , and when two PDFs are closer, the JSD gets smaller. Although no specific changes in the temporal JSD of patient 1 were identified, a drastic change was noted in patient 2 a few days before death. The JSD of vital signs could be useful information, at least in patient 2 , in estimating the last days of the life.

The missing data rate in time-series data is shown in Figures 4 and 5. The missing data rate of patient 1 exponentially increases a few weeks before death and can be an indicator for impending death. For patient 2, however, the missing data rate does not show characteristic changes before death like in patient 1 . Therefore, the missing data rate cannot be a universal indicator for impending death. Table 2 summarizes the applicability of the JSD and missing data rate as signs for predicting death in the two patients.

\section{Discussion}

We have studied the detection of characteristic changes in vital signs and their association with impending death based
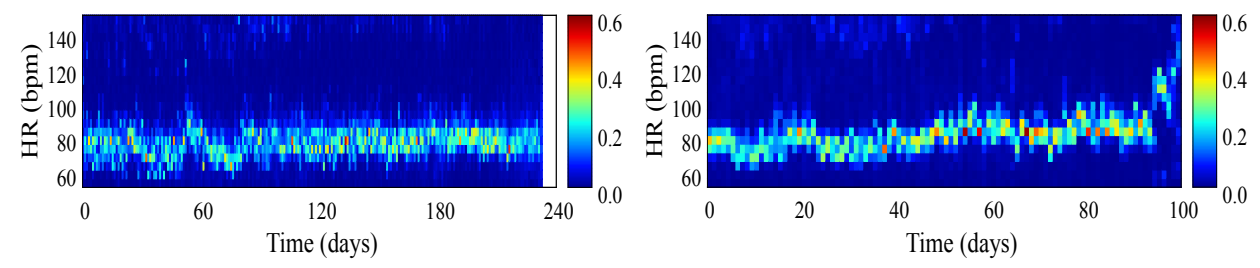

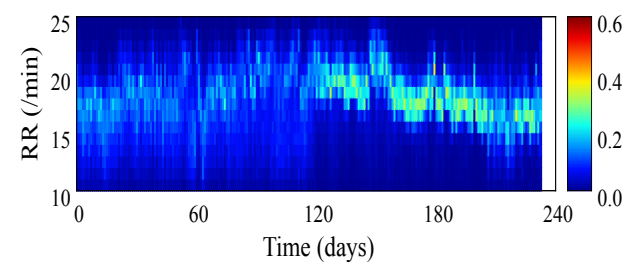

(a) Patient 1

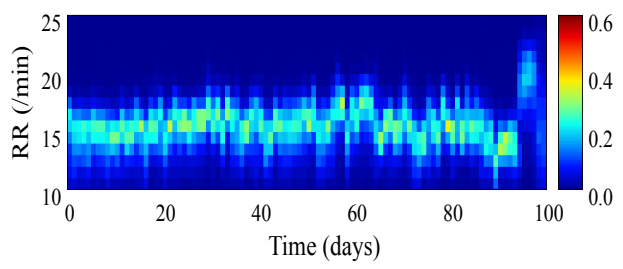

(b) Patient 2

Figure 1: Temporal changes in the PDFs of vital signs. 


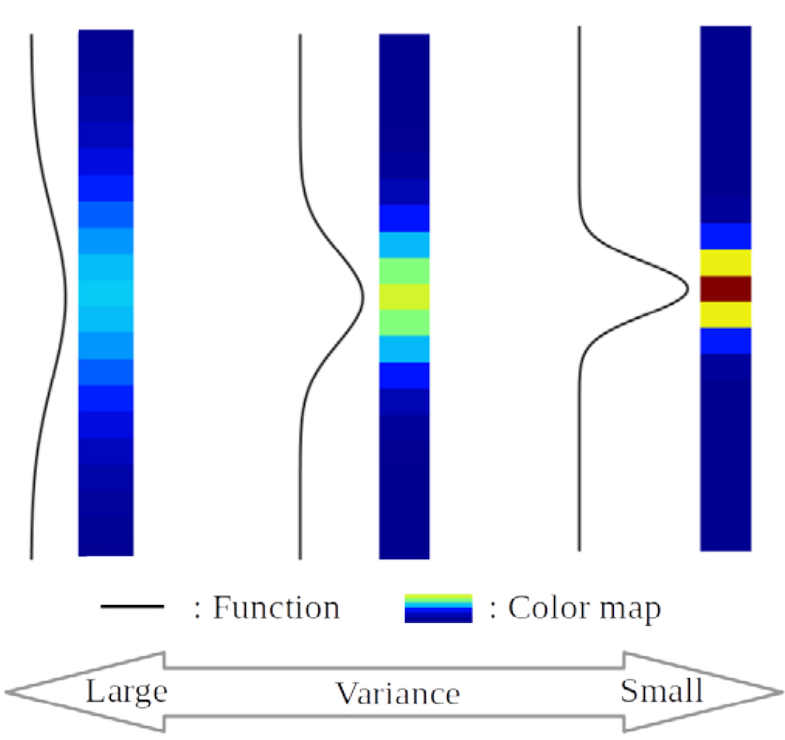

Figure 2: How to interpret the PDFs represented by color maps.
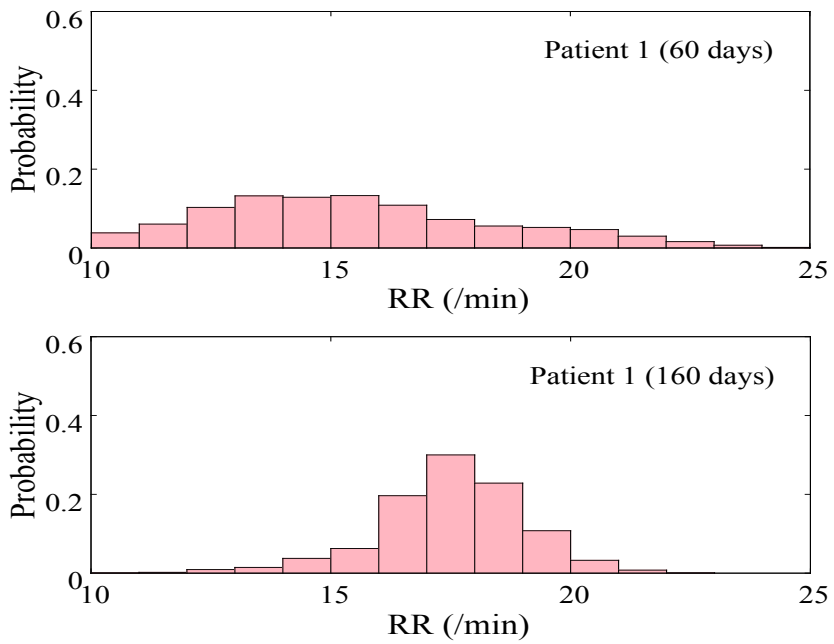

Figure 3: PDFs of the respiratory rate of patient 2 at 60 and 160 days.

on the data measured by the pressure-sensing mat. We found a significant increase in the missing data rate in the final 2 weeks of life for patient 1 and a drastic increase in the JSD in the last 3 days $S$ ec of life for patient 2. However, we could not identify the universal characteristic changes in vital signs related to impending death.

A number of researches studied how vital signs vary at the end of life. To our knowledge, this study is the first attempt to utilize high-temporal-resolution data measured by a less invasive pressure-sensing mat and to analyze large data using a machine learning method to examine the variations in the final days of the patient's life. Although Bruera et al. [4] do not support routine vital sign monitoring of patients who are imminently dying because vital signs cannot be used for prognosis prediction, we believe that it would be acceptable with less invasive sensors such as the pressure-sensing mat used in this study.
However, there are certain limitations in our study. First, the data utilized for the analysis were from only two subjects. Second, we were not able to define the criterion or threshold value to detect the changes associated with impending death because of limitation of data. More data should be analyzed to demonstrate the practicability of the proposed method. Future study needs to investigate the applicability of other sophisticated method for time-series analysis such as seasonal adjustment method [17].

\section{Conclusion}

We have proposed a new methodology to detect specific changes in vital signs that are related to impending death based on the high-temporal-resolution data measured by a pressure-sensing mat and data analysis using density ratio 

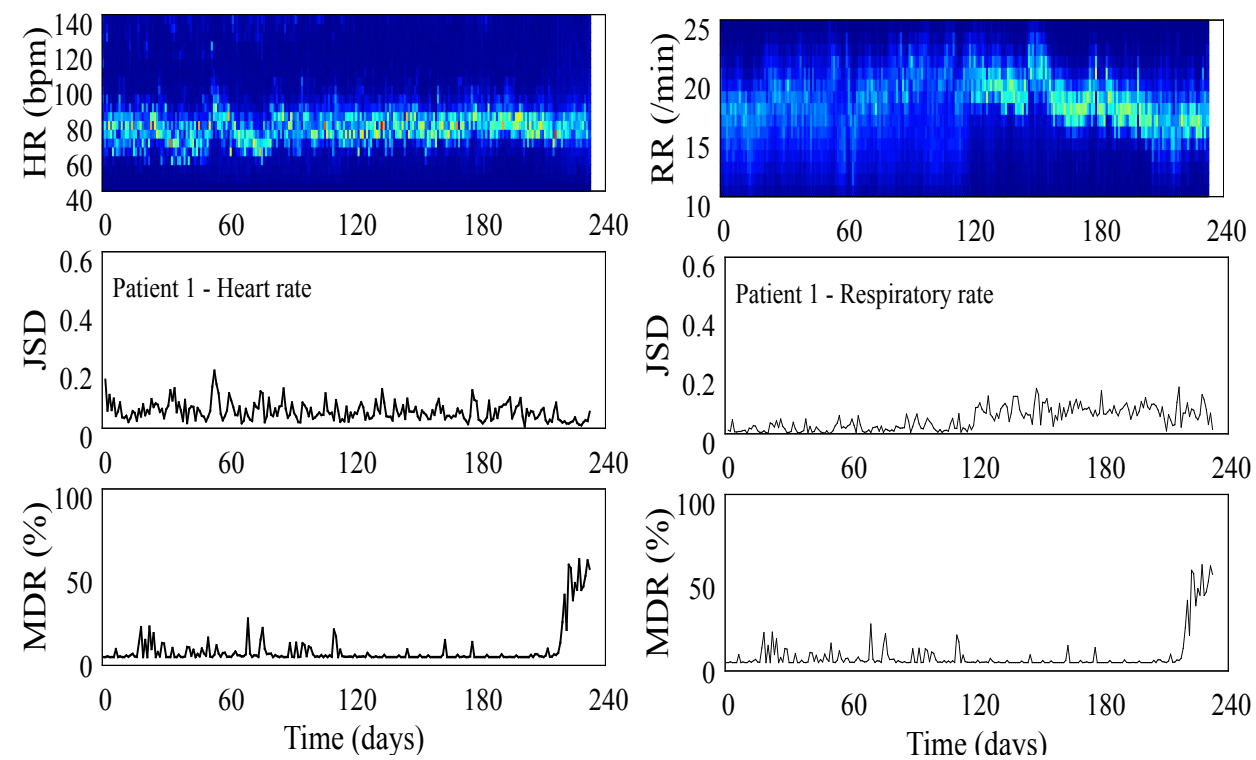

Figure 4: JSD and missing data rate of patient 1.
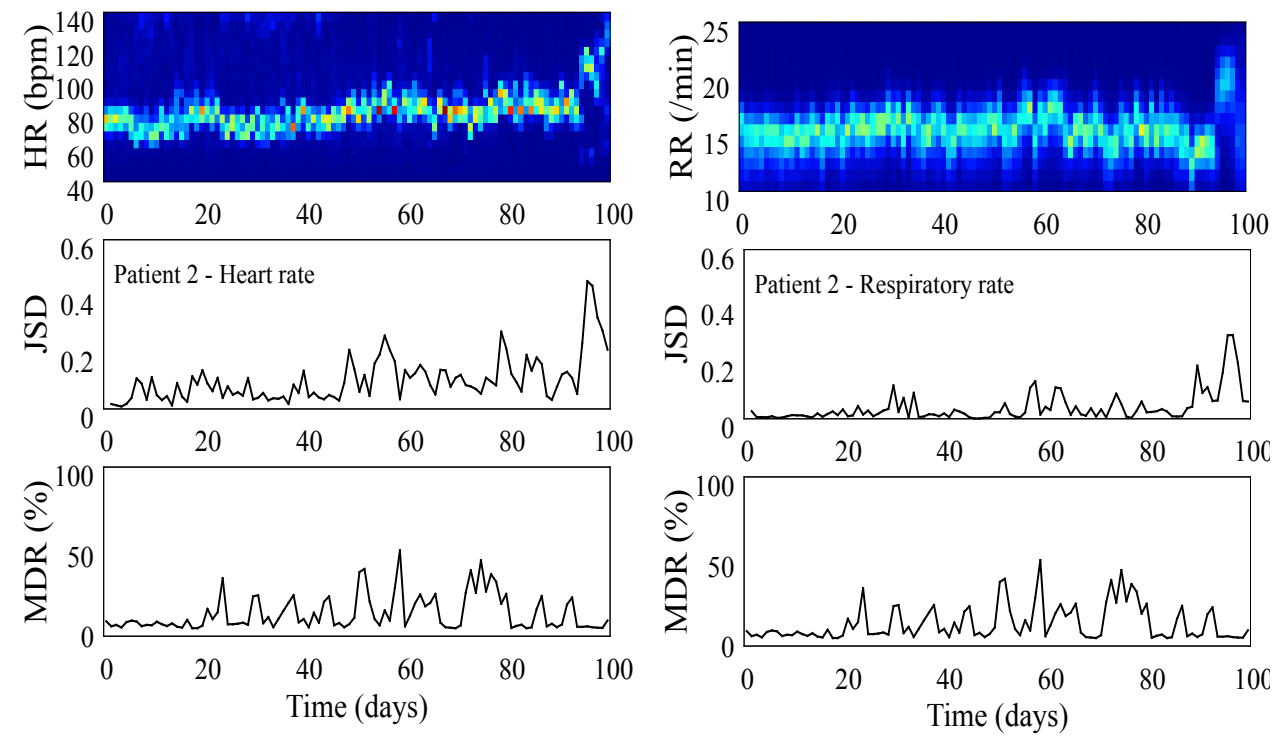

Figure 5: JSD and missing data rate of patient 2.

Table 2: Applicability of JSD and data missing rate as signs for predicting death for two patients.

\begin{tabular}{lll}
\hline & Patient 1 & Patient 2 \\
\hline JSD & Not applicable & Applicable \\
Data missing rate & Applicable & Not applicable \\
\hline
\end{tabular}

\section{Author Contribution}

T. Shuku, N. Sakano and M. Morita have contributed to the conception and design of research; N. Sakano and S. Kasahara collected and managed the research data; T. Shuku and M. Mizuki analyzed the research data; T. Shuku, N. Sakano, M. Morita and S. Kasahara performed the interpretation the data and results. T. Shuku has drafted the article; N. Sakano revised the article. All authors have given approval of the final version to be published.

\section{Conflict of Interest}

The authors declare no conflicts of interest associated with this manuscript. 


\section{References}

[1] Morita T, Ichiki T, Tsunoda J, Inoue S, Chihara S. A prospective study on the dying process in terminally ill cancer patients. Am J Hosp Palliat Care. 1998; 15: 217-222.

[2] Lunney JR, Lynn J, Foley DJ, Lipson S, Guralnik JM. Patterns of functional decline at the end of life. JAMA. 2003; 289: 2387-2392.

[3] Kao YH, Chen CN, Chiang JK, Chen SS, Huang WW. Predicting factors in the last week of survival in elderly patients with terminal cancer: a prospective study in southern Taiwan. J Formos Med Assoc. 2009; 108: 231-239.

[4] Bruera S, Chisholm G, Santos RD, Crovador C, Bruera E, Hui D. Variations in vital signs in the last days of life in patients with advanced cancer. J. Pain Symptom Manage. 2014; 48: 510-517.

[5] Hui D, Santos RD, Chisholm G, Bansal S, Crovador CS, Bruera E. Bedside clinical signs associated with impending death in patients with advanced cancer: preliminary findings of a prospective, longitudinal cohort study. Cancer. 2015; 15 : 960-967.

[6] Saliba D, Elliott M, Rubenstein LZ, Solomon DH, Young RT, Kamberg CJ. The Vulnerable Elders Survey: a tool for identifying vulnerable older people in the community. J Am Geriatr Soc. 2001; 49: 1691-1699.

[7] Porock D, Parker-Oliver D, Petroski GF, Rantz M. The MDS Mortality Risk Index: the evolution of a method for predicting 6-month mortality in nursing home residents. BMC Res Notes. 2010; 3: 200.

[8] Yourman LC, Lee SJ, Schonberg MA, Widera EW, Smith AK. Prognostic indices for older adults: a systematic review. JAMA. 2012; 307: 182-192.

[9] Nishida Y, Takeda M, Mori T, Mizoguchi H, Sato T. Unrestrained and non-invasive monitoring of human's respiration and posture in sleep using pressure sensors. JRSJ. 1998; 16: 705-711.

[10] Watanabe K, Watanabe H. Study on the non-restrictive vital bio-measurement by the air mattress method. SICE. 2000; 36: $894-900$.

[11] Kogure T, Shirakawa S, Shimokawa M, Hosokawa Y. Automatic sleep/wake scoring from body motion in bed: validation of a newly developed sensor placed under a mattress. J Physiol Anthropol. 2011; 30: 103-109.

[12] Pal A, Mukherjee A, Dey S. Future of healthcare sensor data-driven prognosis. In: Prasad R, Dixit S, editors. Wireless world in 2050 and beyond: a window into the future! Springer Series in Wireless Technology. Switzerland: Springer; 2016. p. 93-109.

[13] Sugiyama M, Suzuki T, Kanamori T. Density ratio estimation in machine learning. Cambridge: Cambridge University Press, 2012.

[14] Rakthanmanon T, Campana B, Mueen A, Batista G, Westover B, Zhu Q. Searching and mining trillions of time series subsequences under dynamic time warping. Proceedings of the 18th ACM SIGKDD International Conference on Knowledge Discovery and Data Mining, KDD 12; 2012 Aug 12-16; Beijing, China: ACM; 2012.

[15] Idé T, Inoue K. Knowledge discovery from heterogeneous dynamic systems using change-point correlations. In: Proceedings of 2005 SIAM International Conference on Data Mining (SDM 05), Newport Beach, CA, 571-576, 2005.

[16] Gombay E. Change detection in autoregressive time series. J Multivariate Anal. 2008; 99: 451-464.

[17] Genshiro K. Introduction to time-series modeling. Boca Raton, Florida: CRC Press, 2010. 